\title{
Title:
}

\section{Relationships between adiposity and left ventricular function in adolescents: mediation by blood pressure and other cardiovascular measures}

Abstract: $\max 250$ words.

Introduction Increased adiposity is associated with poorer left ventricular (LV) function but the mediating role of blood pressure (BP) and other cardiovascular measures is unknown. We investigated this in adolescents in a UK birth cohort, the Avon Longitudinal Study of Parents and Children.

Methods Fat and lean mass were assessed by dual energy X-ray absorptiometry (DXA) in 2068 individuals (age 17.7(SD 0.32) years; 45\% male; weight 67(SD 13) $\mathrm{kg}$ ). BP was measured and echocardiography performed. Tissue Doppler systolic (s'), transmitral E/A and (e') were used as measures of LV systolic and diastolic function respectively. Mediation was estimated by structural equation modelling; height, sex, socioeconomic position, lean mass and smoking were included as potential confounders in all models.

Results: Table 1 shows the total and direct (total - indirect (mediated)) effects for each potential mediator, alongside \%mediation. No convincing associations were found between fat mass and s' or e'.

Table 1. Mediation of the association between fat mass and $\mathrm{E} / \mathrm{A}$

\begin{tabular}{lccc} 
Mediator & $\begin{array}{c}\text { Total effect } \\
\text { (standardized } \boldsymbol{\beta} \text { ) }\end{array}$ & $\begin{array}{c}\text { Direct effect } \\
\text { (standardized } \boldsymbol{\beta} \text { ) }\end{array}$ & \% mediation \\
\hline None & $-0.15(95 \% \mathrm{Cl}-0.20,-0.10)$ & & \\
Mean arterial pressure (MAP) & -0.14 & -0.11 & 21 \\
Pulse pressure (PP) & -0.13 & -0.13 & 0 \\
Heart rate (HR) & -0.14 & -0.11 & 21 \\
Total arterial elastance (TAE) & -0.14 & -0.15 & -7 \\
Total peripheral resistance (TPR) & -0.15 & -0.15 & 0 \\
End-diastolic volume (EDV) & -0.15 & -0.17 & -13
\end{tabular}

Conclusions: MAP, HR and EDV, an indicator of preload, are important mediators of the effect of adiposity on diastolic function in adolescence. These findings emphasize the importance of adiposity and risk factor control in adolescence. 